\title{
Case Report \\ Retinal Vascular Tortuosity in a Patient with Weill-Marchesani Syndrome
}

\author{
Kevin Gallagher, ${ }^{1}$ Tahrina Salam, ${ }^{1}$ Barron Sin, ${ }^{2}$ Sandy Gupta, ${ }^{2}$ and Hadi Zambarakji \\ ${ }^{1}$ Department of Ophthalmology, Whipps Cross University Hospital NHS Trust, Whipps Cross Road, Leytonstone, \\ London E11 1NR, UK \\ ${ }^{2}$ Department of Cardiology, Whipps Cross University Hospital NHS Trust, Whipps Cross Road, Leytonstone, \\ London E11 1NR, UK \\ Correspondence should be addressed to Kevin Gallagher, gallagherkr@googlemail.com \\ Received 6 November 2011; Accepted 4 December 2011 \\ Academic Editors: N. Fuse and D. K. Roberts
}

Copyright ( $) 2011$ Kevin Gallagher et al. This is an open access article distributed under the Creative Commons Attribution License, which permits unrestricted use, distribution, and reproduction in any medium, provided the original work is properly cited.

\begin{abstract}
Weill-Marchesani syndrome (WMS) is a rare connective tissue disorder with characteristic phenotypic skeletal and ocular manifestations. A 28-year-old myopic female presented with an 8-month history of bilateral blurred vision. On examination, she was noted to be of short stature with brachydactyly. On ocular examination, she was found to be spherophakic with bilateral inferiorly subluxated lenses. Serum and urine homocysteine were normal and a syphilis screen was negative. A diagnosis of Weill-Marchesani syndrome was made. Fundoscopy revealed bilateral tortuous retinal vessels. We report the first illustrated case of retinal vascular tortuosity as an ocular manifestation of Weill-Marchesani syndrome.
\end{abstract}

\section{Introduction}

Weill-Marchesani syndrome (WMS) is a rare connective tissue disorder with characteristic phenotypic features of short stature, brachycephaly, brachydactyly with limited range of joint movement. Ocular manifestations include microspherophakia with secondary myopia, lens subluxation, and glaucoma secondary to progressive shallowing of the anterior chamber. A review of 128 reported cases of WMS did not describe retinal vascular tortuosity as a feature of the condition [1]. A case series of patients with congenital heart disease identified one patient with WMS with retinal vascular tortuosity (RVT) [2]. We present an illustrated case of retinal vascular tortuosity in WMS in the absence of congenital heart disease.

\section{Case Report}

A 28-year-old female presented with an 8-month history of bilateral blurred vision and progressive myopia. Her parents were first cousins. She had three male siblings, all of whom were healthy with no systemic illnesses. She had no other medical history of note. There was no history of ocular trauma. On examination, she was noted to be of short stature with brachydactyly (Figure 1), broad hands and feet, and limited joint mobility. Visual acuity was $0.9 \log$ MAR in the right eye and $1.0 \log$ MAR in the left. Her most up-todate refraction was as follows: right $-9.25 /-3.00 \times 160$, left $-8.75 /-4.00 \times 40$. She was found to be spherophakic with bilateral inferiorly subluxated lenses (Figure 2). Intraocular pressures were 17 and $18 \mathrm{mmHg}$ right and left. Fundoscopy revealed bilateral tortuous retinal vessels with normal optic discs (Figure 3).

Corneal topography was consistent with keratoconus. Keratometry readings on IOL Master (IOL Master v5, Carl Zeiss Meditech) were right K1 47.01 D K2 49.49 D and left K1 54.00 D and K2 63.08 D. Laboratory investigations revealed normal serum and urine homocysteine and a negative syphilis serology.

The diagnosis of WMS is a clinical one and was made on the basis of short stature and brachydactyly, microspherophakia, ectopia lentis, and the absence of other causes of lens dislocation. While the diagnosis is a clinical one, genetic tests can help confirm the diagnosis. Mutations in the 


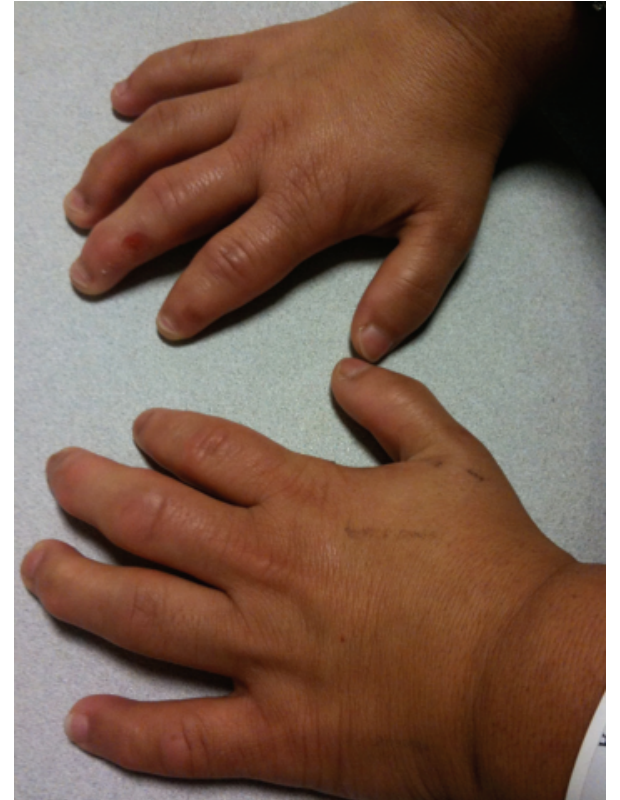

FIGURE 1: Brachydactyly. The patient had limited joint mobility.

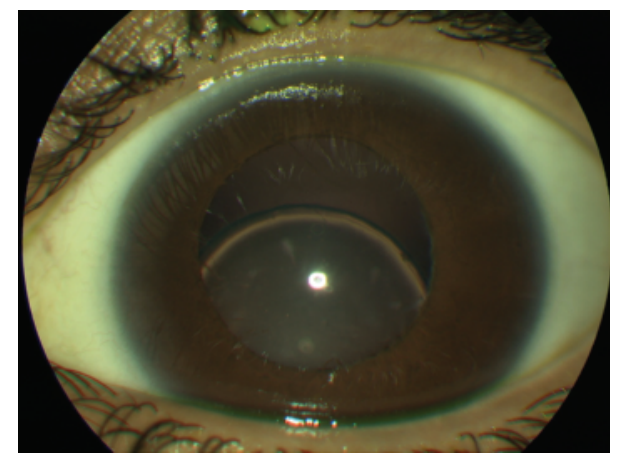

Figure 2: Inferiorly subluxated lens.

fibrillin-1 gene and the ADAMTS10 gene have been found in autosomal dominant and autosomal recessive forms of WMS $[3,4]$. Mutations in the ADAMTS17 gene can give rise to a phenotype resembling WMS [5]. Genetic testing was not performed in this case.

A preoperative cardiology review was requested. Transthoracic echocardiogram has demonstrated normal gross cardiac morphology and good biventricular functions. Mild mitral regurgitation was noted with no haemodynamic consequence.

The patient underwent a pars plana vitrectomy with lensectomy and Artisan anterior chamber intraocular lens (Abbot Medical Optics, Ill, USA) implantation bilaterally. Postoperative visual acuity was $0.24 \log$ MAR right and $0.30 \log$ MAR left.

\section{Discussion}

We report the first illustrated case of retinal vascular tortuosity associated with WMS, in the absence of congenital

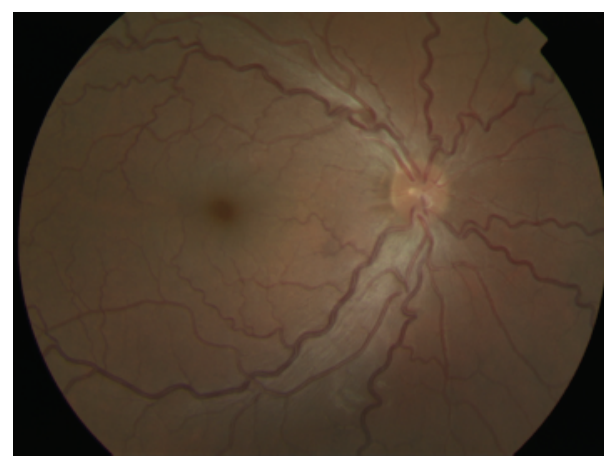

FIgURE 3: Retinal vascular tortuosity with normal optic discs.

heart disease. The association of RVT with WMS has been described in a case series of 240 patients with cyanotic, obstructive, or volume-overloading congenital heart disease [2]. In this series, RVT was strongly correlated with hypoxia and a low haematocrit. Other series of patients with cyanotic congenital heart disease have found an association between RVT and polycythaemia [6, 7]. This finding is supported by the observation that RVT improves with the correction of the cardiac defect and subsequent normalisation of the haematocrit [6]. The patient in this report had no echocardiographic indication of cyanotic, obstructive, or volume-overloading congenital heart disease, was not hypoxic, and had a normal haematocrit. Thus, the retinal vascular changes observed in this case of WMS are likely to represent a different clinical entity to that associated with cyanotic congenital heart disease.

\section{References}

[1] L. Faivre, H. Dollfus, S. Lyonnet et al., "Clinical homogeneity and genetic heterogeneity in Weill-Marchesani syndrome," American Journal of Medical Genetics, vol. 123, no. 2, pp. 204207, 2003.

[2] A. M. Mansour, F. F. Bitar, E. I. Traboulsi et al., "Ocular pathology in congenital heart disease," Eye, vol. 19, no. 1, pp. 29-34, 2005.

[3] L. Faivre, R. J. Gorlin, M. K. Wirtz et al., "In frame fibrillin1 gene deletion in autosomal dominant Weill-Marchesani syndrome," Journal of Medical Genetics, vol. 40, no. 1, pp. 34-36, 2003.

[4] N. Dagoneau, C. Benoist-Lasselin, C. Huber et al., "ADAMTS10 mutations in autosomal recessive weill-marchesani syndrome," American Journal of Human Genetics, vol. 75, no. 5, pp. 801806, 2004.

[5] J. Morales, L. Al-Sharif, D. S. Khalil et al., "Homozygous mutations in ADAMTS10 and ADAMTS17 cause lenticular myopia, ectopia lentis, glaucoma, spherophakia, and short stature," American Journal of Human Genetics, vol. 85, no. 5, pp. 558-568, 2009.

[6] E. M. Kohner, E. M. Allen, K. B. Saunders, V. M. Emery, and C. Pallis, "Electroencephalogram and retinal vessels in congenital cyanotic heart disease before and after surgery," British Medical Journal, vol. 4, no. 573, pp. 207-210, 1967.

[7] I. Tsui, K. Shamsa, J. K. Perloff, E. Lee, R. S. Wirthlin, and S. D. Schwartz, "Retinal vascular patterns in adults with cyanotic congenital heart disease," Seminars in Ophthalmology, vol. 24, no. 6, pp. 262-265, 2009. 


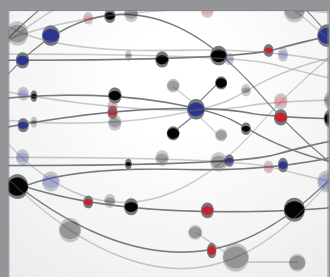

The Scientific World Journal
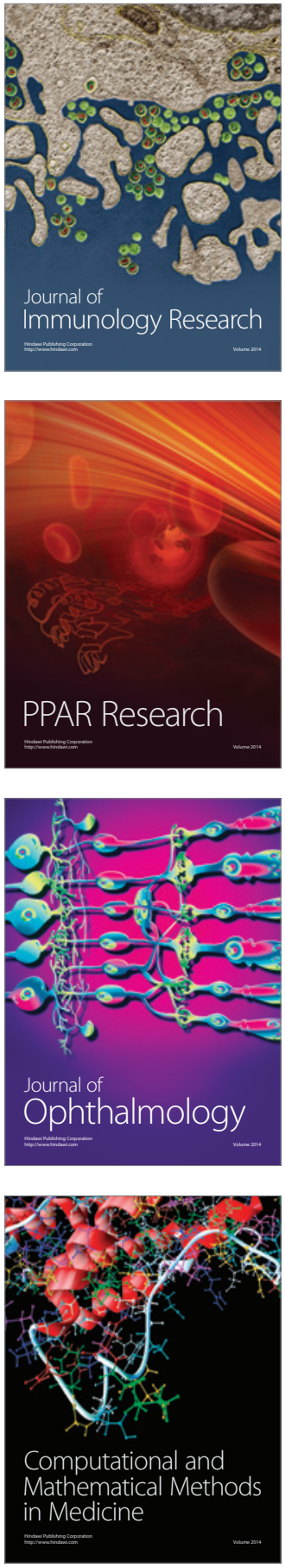

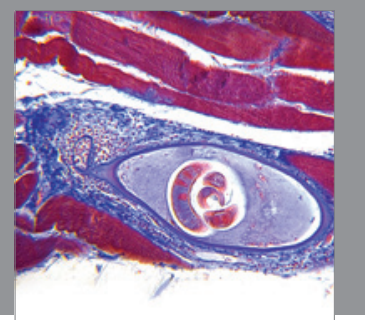

Gastroenterology

Research and Practice
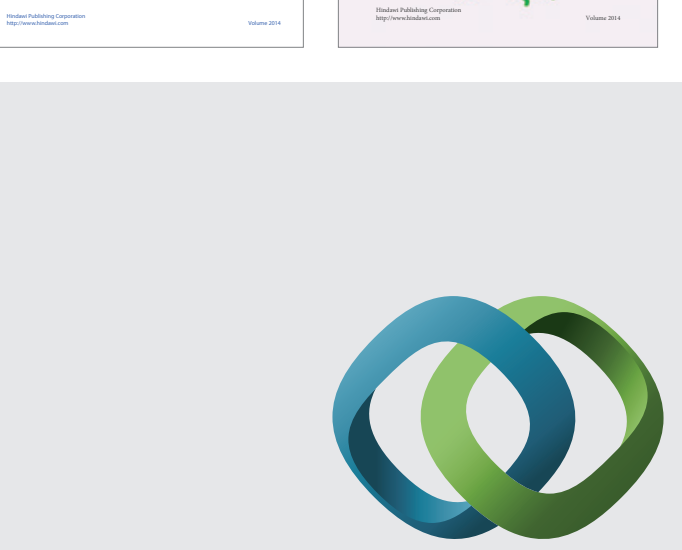

\section{Hindawi}

Submit your manuscripts at

http://www.hindawi.com
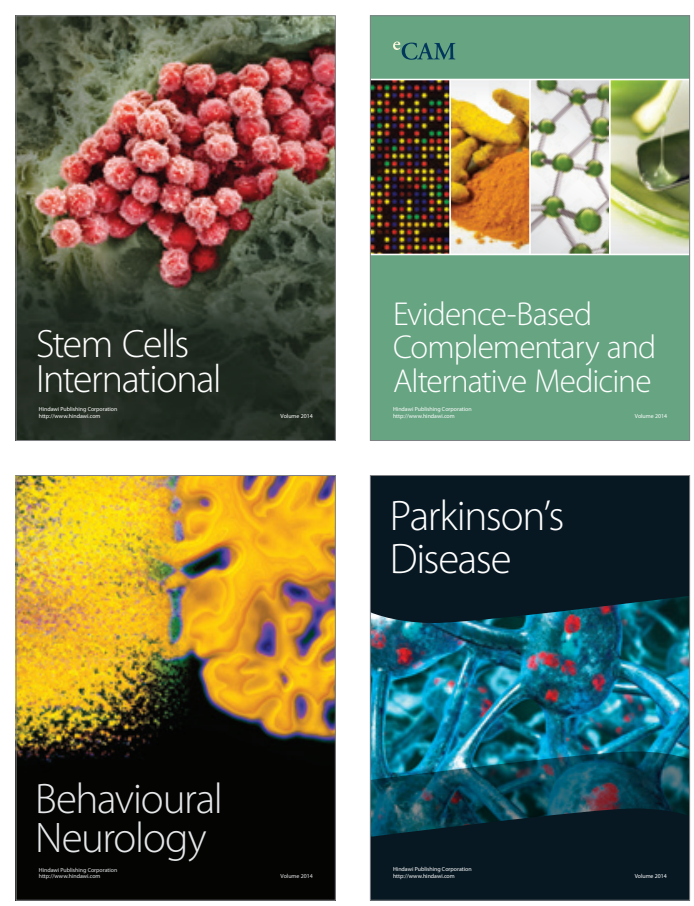

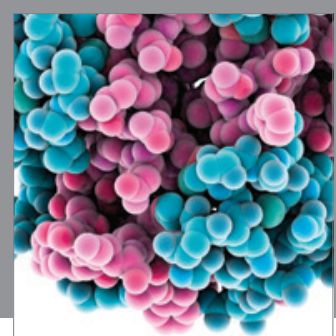

Journal of
Diabetes Research

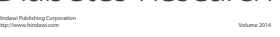

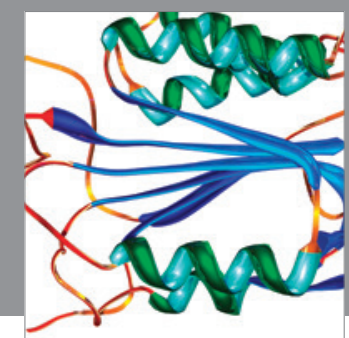

Disease Markers
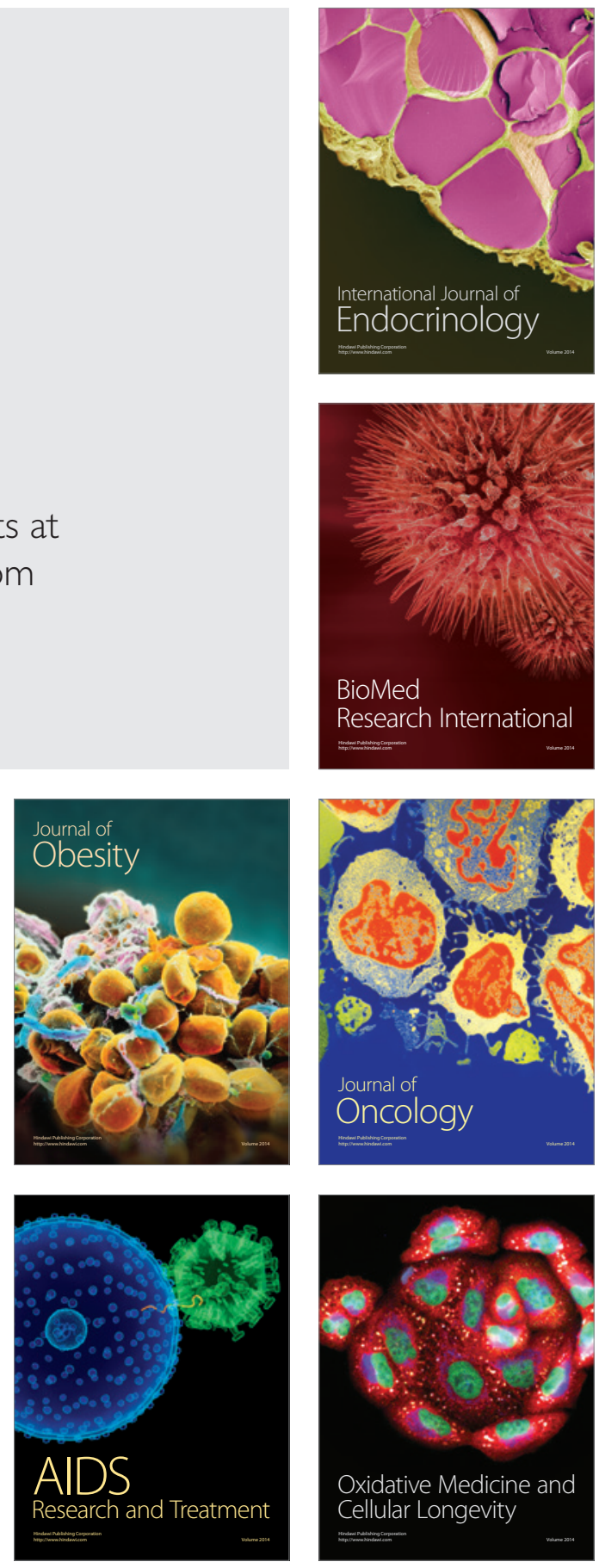